\title{
A Comparison Study of Simulation-Based Prediction Tools for Air Temperature and Outdoor Thermal Comfort in a Tropical Climate
}

\author{
Elif Esra Aydin ${ }^{1}$, J. Alstan Jakubiec ${ }^{2}$, Steve Kardinal Jusuf ${ }^{3}$ \\ ${ }^{1}$ Singapore University of Technology and Design, Singapore \\ ${ }^{2}$ University of Toronto, Toronto, Canada \\ ${ }^{3}$ Singapore Institute of Technology, Singapore
}

\begin{abstract}
Predicting the urban microclimate using simulation tools can help designers to increase outdoor thermal comfort. This study discusses 4 prediction tools from an urban designer's view regarding their capabilities, limitations, ease of use, and accuracy. ENVI-met, RayMan, SOLWEIG, and STEVE are urban microclimate prediction tools investigated in this study. Measured and simulated air temperature, mean radiant temperature and thermal comfort indices are compared across multiple sites and weather conditions. The novelty is evaluating 4 tools' ability to help drive design decisions and the predictive accuracy of the tools that may influence those decisions from the view of an urban designer.
\end{abstract}

\section{Introduction}

Urban Heat Island (UHI) is the differentiation of ambient air temperature between urban and rural area settlements caused by microclimatic changes in cities. The Intergovernmental Panel on Climate Change (IPCC) (IPCC Report, 2018) reported a $1.5^{\circ} \mathrm{C}$ increment in urban air temperatures that demonstrates the need of UHI mitigation actions as part of addressing the global warming challenge.

Regarding the role of cities on UHI, rapidly unrestrained developing cities cause loss of greenery, increasing built area with landscape being replaced by impermeable surfaces on the ground, and increasing the thermal mass and decreasing albedo of built environment via construction material of the façade designed. These trends in the built environment result in UHI increasing heat stress and decreasing thermal comfort in cities worldwide. One way to ameliorate the UHI phenomenon may be to design the urban context with the help of computational tools that can evaluate the microclimatic impacts of urban design for thermal comfort.

In the past two decades, researchers in the fields of climatology, urban planning, and meteorology science have been developing microclimate prediction tools such as SOLENE (Miguet et al, 1996), ENVI-met (Bruse and Fleer, 1998), RayMan (Matzarakis et al, 2007), SOLWEIG (Lindberg et al, 2008), STEVE (Jusuf and Wong, 2009), and CityComfort+ (Huang et al, 2014). The challenge to address UHI has led researchers to integrate various specialties for 'urban design' issues which integrates urban planners, meteorologists, scientists, practitioners, and designers. Regarding the integration potential, urban designers should know how to use the prediction tools in order to bring awareness of the impact of their designs on UHI. Therefore, this study presents an evaluation of environmental predictor tools from a designer or planner's point of view-assessing their ease of use, capabilities, limitations, and accuracy. For the tropical climate in Singapore, four tools in the literature were selected: ENVI-met, SOLWEIG, RayMan, and STEVE. The previous studies in the literature seeking the comparison of the selected tools in the sense of accuracy on Tmrt prediction methods (Szucs et al., 2014; Chen et al., 2014). However, while Ozkeresteci et al. (2003) focused on ENVI-met tool, the study of Wong et al. (2012) was a more detailed comparison about the tools' ability but only between the ENVI-met and STEVE tools. The authors therefore intend for this study to contribute a broader knowledge of the usability of the prediction tools on thermal comfort and UHI mitigations for future practitioners, designers, and planners.

This study addresses the following research questions: What are the impacts of limitations on the accuracy of tools considering the comparison with measured environmental data? What are the similarities and differences of the primary inputs and input parameters for each tool? What are the advantages and disadvantages of each tool when being used throughout an urban design process? To respond to the research questions mentioned above, the study was structured for site experiments within practical process completed by a validity check that is explicitly explained in 'methodology' section.

\section{Methodology}

Considering the scope of the study, this project workflow covers 3 phases: (1) field study, (2) simulation, (3) validation of simulation tools. The first step is a field study phase where data was collected for two weeks per each location point with four data loggers in a university campus. Passage zone (P), Courtyard zone (C), Greenery zone $(G)$ were selected to represent three different built environment contexts for 12 measurement points. In parallel to the field study, the simulation phase is to model the university campus area while assessing of ease-of-use, capabilities, and tool interfaces of four acknowledged microclimate prediction tools: ENVI-met, RayMan, SOLWEIG, STEVE tool. Lastly, the validation phase is a comparison of the measured data with simulation results to evaluate the accuracy of tools on air temperature (Ta), mean radiant temperature (Tmrt: Mean Radiant 
Temperature) and Physiologically Equivalent Temperature (PET), which is calculated using inputs of Ta, Tmrt, air velocity, relative humidity, physiological factors and clothing factors.

\section{Field Study Phase}

\section{Geographic Location of Study Site:}

The chosen case site is a university campus in Singapore - a hot and humid tropical city located near the equator. . The field study measurement has been recorded during June and July, a monsoonal season where there is a slight air temperature reduction (Meteorological Service Singapore, 2018). The positioning of 12 measurement points in 3 different zones of the campus site aims to increase the sample points for data and to understand the correlation of UHI with building massing, greenery, and pavement variety. Figure 1 illustrates the locations for each measurement point in the campus area on the plan, and Figure 2 (left column) contains images of each measurement location.

\section{Location Points:}

The first zone is located in a canyon between two buildings and is an open passage area; the second location is a semi-open courtyard zone bounded on three sides; lastly, the third location, is a grassy area with a building only on the west side (Figure 1, Figure 2). These locations were chosen in order to study the varied impact of buildings, greenery, and pavement on the changing microclimate and the accuracy of microclimate prediction tools. The passage zone $(\mathrm{P})$ is predominantly surrounded by buildings while the courtyard zone $(\mathrm{C})$ has a balance between greenery and buildings. The greenery zone $(\mathrm{G})$ located close to a building is predominantly covered by grassy land and trees.

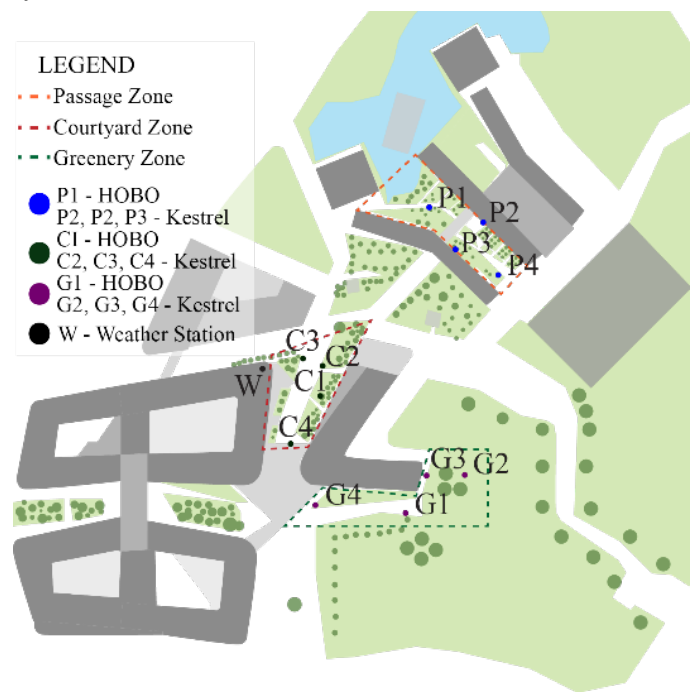

Figure 1: Measurement points on plan scheme.

\section{Period of Data Collection:}

Meteorological data was collected during a two-week period at each location point using a 5-minute time interval. The duration of data collection was therefore 6 weeks in total (two weeks for each zone) between $22^{\text {nd }}$ June 2018 and $27^{\text {th }}$ July 2018. However, the data used in analysis was a three-day sample per each location point selected to correspond to mainly overcast, intermediate and clear sky conditions. Table 1 illustrates the measurement dates and weather conditions for each point by given cloud cover values for simulation tools.

Table 1: Weather condition of data collection duration.

\begin{tabular}{llll}
\hline Location Zone & Date & $\begin{array}{l}\text { Sky } \\
\text { Condition }\end{array}$ & $\begin{array}{l}\text { Cloud } \\
\text { Cover } \\
\text { (oktas) }\end{array}$ \\
\hline Passage Zone & 22.06 .2018 & Intermediate & 4 \\
(P) & 26.06 .2018 & Overcast & 8 \\
& 28.06 .2018 & Clear & 0 \\
\hline Courtyard Zone & 03.07 .2018 & Overcast & 8 \\
(C) & 06.07 .2018 & Clear & 0 \\
\hline \multirow{2}{*}{ Greenery Zone } & 09.07 .2018 & Intermediate & 4 \\
(G) & 17.07 .2018 & Overcast & 8 \\
& 23.07 .2018 & Intermediate & 4 \\
\hline
\end{tabular}

\section{Measurement Tools:}

Four data loggers - 3 Kestrel Heat Stress Trackers (model 5400) and 1 HOBO Temperature and Humidity Data Logger-were located in each zone. Moreover, an existing weather station of the university campus located at rooftop on a building as seen in Figure 1 has been used for the collection of global horizontal solar radiation data. The heat stress trackers are capable of measuring the ambient air temperature, relative humidity, globe temperature (black globe), and wind velocity while the temperature tool records the ambient air temperature, relative humidity and globe temperature (grey globe). The HOBO sensor is instrumented at a varying height of urban furniture in the campus area, and the Kestrel sensors are fixed at a $1.1 \mathrm{~m}$ height above the ground on tripods (Mayer and Höppe, 1987). Figure 2 illustrates the measurement tools used for this study (right column) while identifying sensors locations for the three instrumentation zones on the left column.

\section{Simulation Phase}

Simulation Tools:

ENVI-met (Bruse and Fleer, 1998), RayMan (Matzarakis et al., 2007), SOLWEIG (Lindberg et al., 2008), and STEVE (Jusuf and Wong, 2009) are microclimate prediction tools used in this study with the aim of clarifying their capabilities, limitations, ease of use and accuracy.

ENVI-met is developed as a surface-plant-air interactionbased microclimate and air quality model. ENVI-met is a "three-dimensional and non-hydrostatic model" which is one of the pioneer tools of microclimate prediction based on wind-driven analysis (Bruse and Fleer, 1998). ENVImet is capable of wind analysis using computational fluid dynamics and environmental pollution analysis besides the prediction of air temperature, Tmrt, and thermal comfort indices such as PET, Predicted Mean Vote (PMV), Standard Effective Temperature (SET*), and Universal Thermal Climate Index (UTCI).

Matzarakis et al. (2007) developed RayMan as an estimation tool for urban climatology which predicts a thermal climate considering human-biometeorology. 
RayMan is a physics-based prediction tool that is capable of predicting Tmrt, SVF, global radiation, and some thermal indices such as PMV, PET, SET*, UTCI, and Perceived Temperature (PT) for a point of interest. Inputs are meteorological data of location, human factors, topography, urban context (building, and trees), and sky view factor (SVF) imagery.

SOLWEIG is a "non-stationary model that is able to calculate the spatial variations of Tmrt" (Lindberg et al., 2015). The tool converts a Digital Surface Model (DSM) to an urban form by using a dataset of elevation values for each grid cell of the whole 3D model. Required inputs are direct, solar radiations (direct, diffuse and global shortwave), air temperature, relative humidity and geographical information of the location (Lindberg et al., 2008). The tool calculates daily shading maps, Tmrt, SVF, and some thermal indices (PET, UTCI). The last version of SOLWEIG has been integrated as a processor into Urban Multiscale Environmental Predictor (UMEP) via QGIS (Lindberg et al., 2015). In this study, SOLWEIG 2015 version has been used by inputting Digital Surface Model (DSM) representations of the campus shown in Figure 1.

The STEVE "Screening Tool for Estate Environment Evaluation" tool is a statistics-based air temperature prediction tool that aims to provide a bridge between research findings and urban planning (Wong et al., 2012). The STEVE tool is a regression model calculating solely ambient air temperature and SVF by using albedo values, solar radiation, and urban context elements (building, pavement, greenery) (Jusuf and Wong, 2009). The former version of STEVE tool is a web-based application, afterward, the tool has been integrated into $3 \mathrm{D}$ model interfaces such as Sketch-up. This study used the published formula of STEVE tool to run microclimate predictions in the Grasshopper canvas for the Rhinoceros 3D modelling platform.

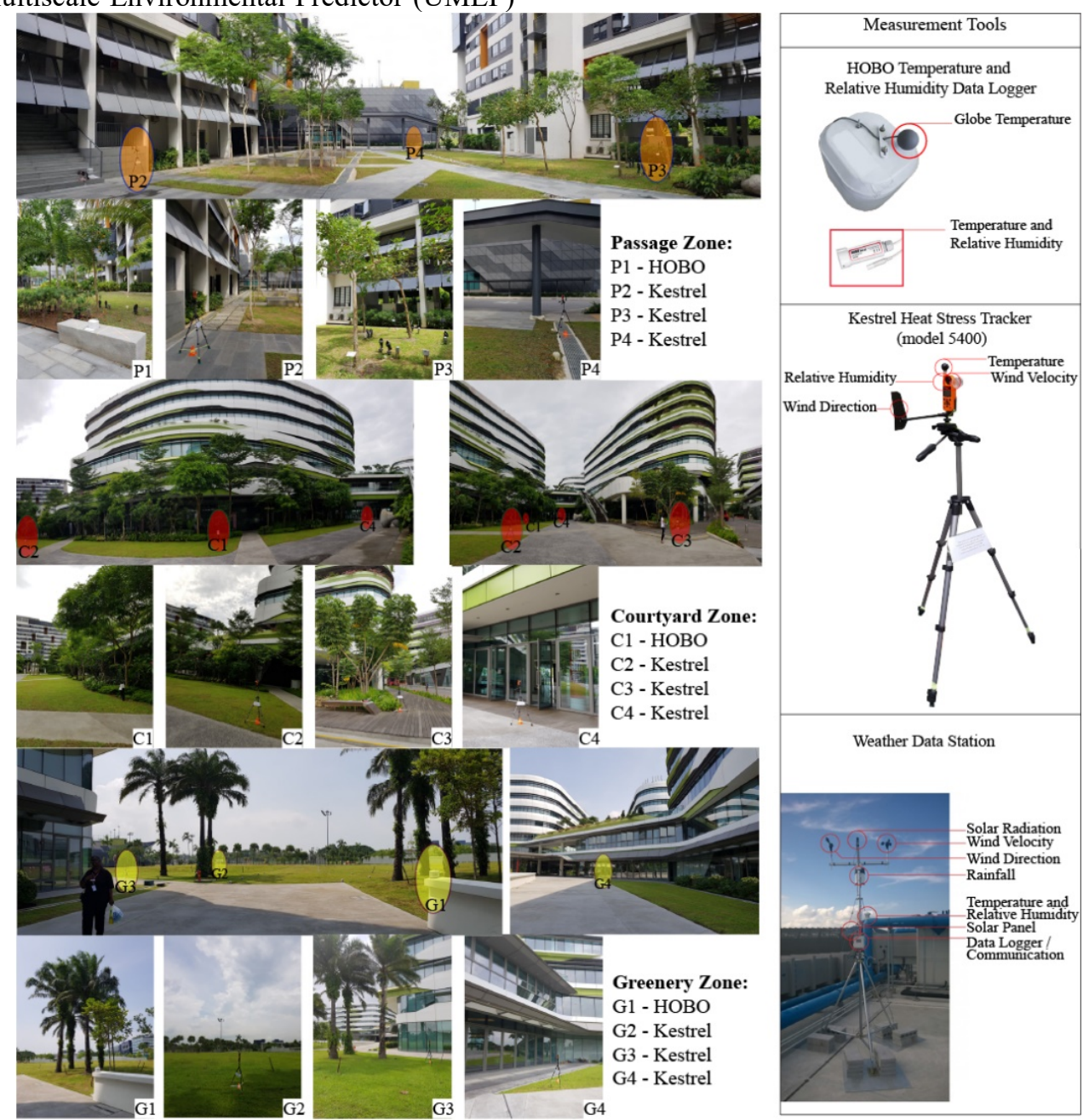

Figure 2: Left Column: Measurement location zones and points; Right Column: Measurement Tools.

\section{Simulation Settings:}

The meteorological and simulation settings exist for each tool as a necessary input for urban microclimate simulations. The geographical data of Singapore $\left(103^{\circ} 59^{\prime} \mathrm{E}, 1^{\circ} 22^{\prime} \mathrm{N}, 16 \mathrm{~m}\right.$ altitude, +8 UTC) (EPW, $2018)$, an assumption for a female profile $(1.58 \mathrm{~m}, 48 \mathrm{~kg}$, 26-age), the standard metabolic activity rate of a standing person (80 W) (Höppe, 1999), and summer clothing $(0.5$ clo) (ASHRAE 55, 2004) have been used as the inputs to all tools. Material details of simulations are assumed to be as follows: 0.3 albedo of surroundings, 0.95 emissivity, and 1.0 Bowen ratio (Matzarakis, 2007). Another assumption is that the terrain model of the simulated site is modelled as flat 
despite the fact that it is slightly inclined. The other setting is grid cell size of the spatial resolution (in $\mathrm{x}$ and y direction width values), which is $1 \mathrm{~m}$ by $1 \mathrm{~m}$ for RayMan and SOLWEIG and $2 \mathrm{~m}$ by $2 \mathrm{~m}$ for ENVI-met. The grid sizes defined give information about the detail level of the urban model drawn into tools. $1 \mathrm{~m} \mathrm{x} 1 \mathrm{~m}$ grid is more detailed due to less abstraction of model forms.

\section{Results and Discussion}

'Results and Discussion' section projects the selected tools capabilities, limitations, ease of use and accuracy rather than presenting 'the best' tool for designers.

\section{Ease of Use}

This section discusses the usability easiness initially the physics-based models and then regression-based models which are evaluated from the point of view of an author of this paper as an architect/researcher who is neither an urban planner nor a meteorology specialist. This evaluation aims to understand the process of simulating a microscale urban analysis using each of the prediction tools considering the complexity of data entry representing the urban context, generating results, and parsing them. Regarding this aim, Table 2 depicts a high-level summary of the comparison of the selected tools based on their interfaces, inputs, and outputs.

Each prediction tool has a different interface to model urban contexts such as grid-based, pixel-based points or a Digital Surface Model (DSM). Regarding the whole simulation process, the modelling of urban context is an initial difficulty faced due to required pre-knowledge for a different format of geometrical modelling and some supportive tools. For instance, SOLWEIG requires the DSM format physical modelling which is not a common format for a designer to create a new design rather than using the existing urban areas accessible by the help of such tools (i.e. ArcGIS and QGIS). For RayMan, shapefile format is a method which is not either a common format for modelling used instead of manual data entry. Thus, modelling the urban form might take more time than tool utilization because of learning various format of physical modelling and the supportive tools by non-expert researchers for urban planning field who do not know how to create these formats. Equally, the manual drawing takes more time to model the urban form correctly regarding the scaling issue in the tools. For instance, due to RayMan needs a pixel-based drawing with 8 points ( 4 points for the bottom, 4 points for the top of the building) for each building, manual modelling and editing complex urban models is difficult and time-consuming. ENVI-met's grid-based format is more straightforward to draw the regular forms except for there is no opportunity to convert an existing model to grid-based format. On the other hand, ENVI-met provides an option to edit the model in both ' $2.5 \mathrm{D}$ ' and 3D. Herein, 2.5D means that each grid cell has one upper and one lower z-height elevation value for any urban element. Although, complex 3D forms are hard to model in ENVI-met due to modelling and editing interface screens (Huttner and Bruse, 2009), nevertheless, 3D edit is one of the significant distinctions of ENVI-met among the microclimate prediction tools (Table 2)

Data entry is another crucial process to specify user satisfaction beside modelling and simulation speed. Apart from the common geographical, temporal and meteorological data inputs summarized in Table 2, ENVI-met requires more effort to provide some specific data such as soil profile and high atmospheric humidity level that can be calculated and estimated by the help of Atmospheric Correction Parameter Calculator (NASA ACPC, 2019). Considering urban fabric's materiality data entry, reflectivity (albedo) and emissivity values can be given separately for each building for RayMan and STEVE tool while SOLWEIG requires one average value for all wall and ground surfaces that are important to provide the accurate environment.

The speed criteria were evaluated the simulation time while taking account of the capability for the amount of day input one at a time. As a result, RayMan is the fastest tool which runs hourly data of 3 days (72 hours) simulations in approximately 2-3 minutes, while ENVImet is the slowest one that runs 48 -hour time interval simulation in about 24-40 hours within all tools. SOLWEIG and the STEVE tool run a day hourly data in approx. 5-7 minutes, however, SOLWEIG model area given was one-third of the STEVE tool's model area given at once.

\section{Physics Calculation Capabilities}

As Table 2 summarizes the outputs, Tmrt, PET and UTCI are the common outputs for ENVI-met, SOLWEIG, and RayMan while the air temperature is common output for ENVI-met and the STEVE tool.

ENVI-met's calculation and output capabilities are substantially different compared to the other tools in this study. Besides the analysis of pollution dispersion, and common outputs, CFD-based wind-driven analysis is the key feature of ENVI-met to acquire wind velocity and pressure maps for the urban model. Moreover, ENVI-met provides soil modelling including heat storage, evaporation, and transpiration with various options like water, grass, earth or pavement and 3D vegetations to represent the urban context accurately.

RayMan estimates thermal comfort and Tmrt, heat and cold stress for a point of interest with the inputs of air temperature, humidity, wind speed and radiation. Also, RayMan can estimate SVF via two ways: drawing the urban context manually and converting a real image to fish-eye image (Nastos et al., 2017).

SOLWEIG diverges from ENVI-met and RayMan by providing the 6-direction longwave and shortwave radiation results for point of interest. SOLWEIG is capable of predicting diffuse and direct radiation when the data of global radiation, air temperature, relative humidity wind velocity, and direction are provided. Regarding the detail of solar calculations of SOLWEIG, the shadow pattern analysis is a unique optional output. As different than RayMan, SOLWEIG can estimate SVF by using 6 direction real images of an existing 
environment for a point of interest: 4 cardinal directions, 1 upward, and 1 downward (Lindberg et al., 2015).

As opposed to previous physics-based models, the STEVE tool is an application of a regression model derived from the measurement of environmental data recorded in Singapore for 3 years period. This model predicts the air temperature for maximum (Tmax), average (Tavg) and minimum (Tmin) values by using a regressed equation based on urban form, the density of greenery, materiality (albedo), temperature, relative humidity, SVF, and solar radiation daily maximum and total daily inputs. While the Tmax regression model uses the daily maximum solar radiation value and the Tavg uses the daily total solar radiation value, the Tmin regression model does not contain solar radiation value likely because it occurs at night (Jusuf and Wong, 2009). A limitation of STEVE is that its regression model is based on data measured in Singapore, so while it is appropriate to this study, it is unlikely that it can be extended to other climate types.

Table 2: Capability Comparison Chart of Microclimate Prediction Tools $*^{*} x$ indicates the presence of the parameter).

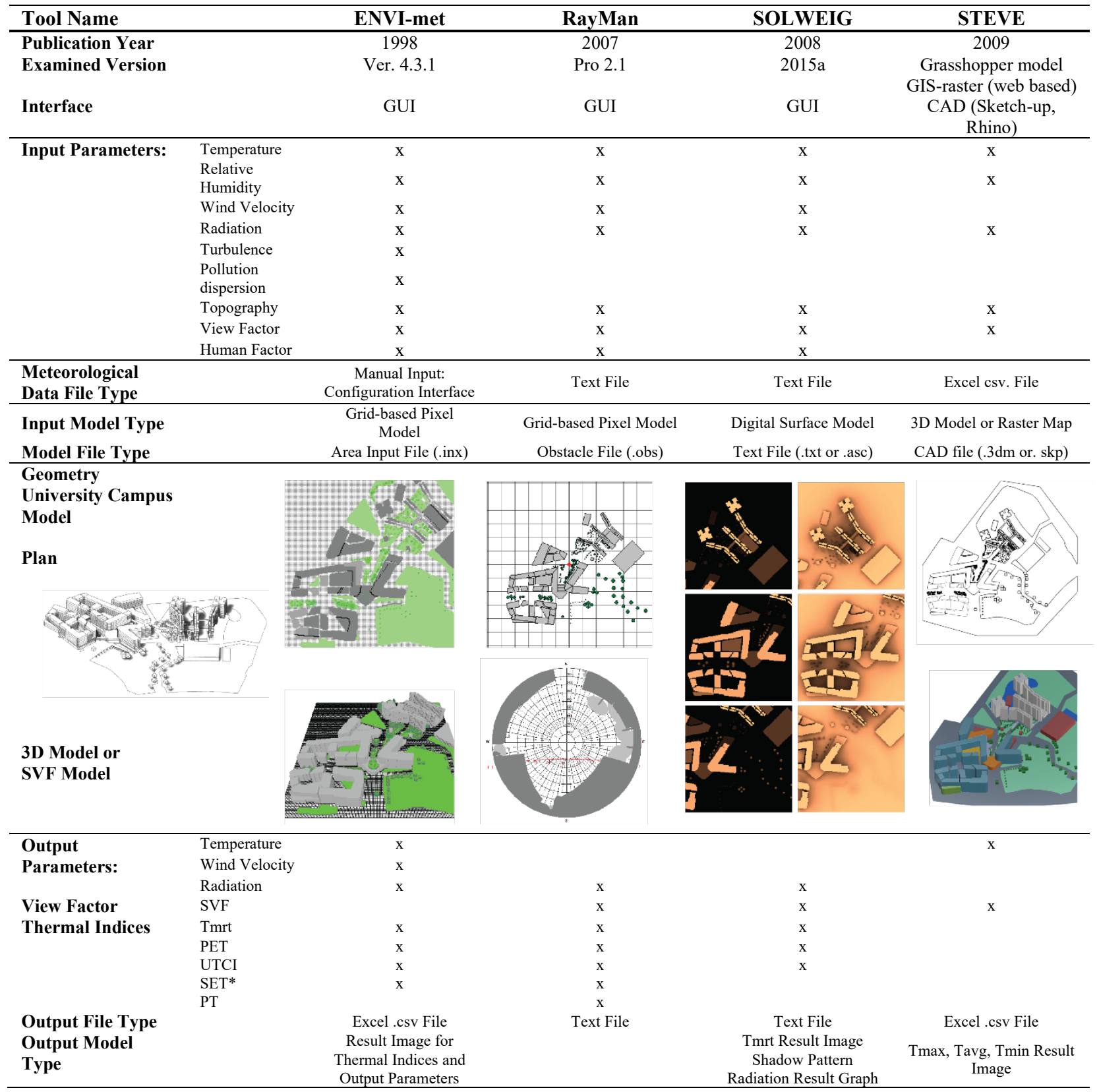

\section{Other Limitations}

A limitation for all tools evaluated is the necessity of simplification on the urban model that might cause the discrepancies on accuracy. SOLWEIG and the STEVE tool have a shortcoming to draw the porous form of the buildings while RayMan does not allow to model a form rather than a rectangle shape building. Also, ENVI-met has a limited grid resolution to model the thin elements such as shading devices on the streets. Due to the oversimplification, the complex forms have to be separated into two or three buildings which might mislead the calculations. 
RayMan is the only tool that is capable of running for three days meteorological data input at once. While SOLWEIG needs one day run because of the daily shadow pattern analysis, STEVE can be set for three-day data together, however specifically solar radiation data of three different days could mislead the input values in the regression formula. ENVI-met, on the other hand, has 24 or 48-hours time interval simulation one at a time (Ozkeresteci et al., 2003). Moreover, RayMan and SOLWEIG can only generate results for a single point of interest at a time, while ENVI-met and STEVE both allow spatial results across a grid of points of the urban model.

Hourly measured wind data is input in SOLWEIG and RayMan for point of interest, however, ENVI-met calculates differing spatial urban wind velocities based on a constant source wind velocity while the standard deviation of our measured data is 0.23 to $1.14 \mathrm{~ms}^{-1}$. Ignatius et al. (2015) integrated a limited (only valid for Tmax) wind model into STEVE tool regression model; however, for the STEVE model examined, the wind data has an assumption that the formula is valid for a calm and clear sky condition (wind velocity below $3 \mathrm{~ms}^{-1}$ ).

On the other hand, anthropogenic heat release and thermal indices prediction are currently out of the scope of the STEVE tool (Jusuf and Wong, 2009).

\section{Accuracy-Comparisons Between Simulated and Measured Data}

The accuracy of tools has been examined particularly with air temperature $(\mathrm{Ta})$, and mean radiant temperature (Tmrt) (Wessel, 2001) as they are some of the most significant variables by affecting human outdoor thermal comfort (Lindberg et al., 2008) and are commonly predicted by most tools assessed in this study. Physiological equivalent temperature (PET) (Höppe, 1999) is calculated to assist in the comparison between SOLWEIG and RayMan. Tmrt is derivable output for ENVI-met, SOLWEIG and RayMan for each point of interest. Scatter plots and line charts illustrate the linear correlation of executed simulation data with the measured data to explain the accuracy via Root Mean Squared Error (RMSE) and Mean Bias Error (MBE) on Figure 3.

This study presents a Tmrt comparison depending on 3 sky conditions defined previously regardless of the difference of the location zones of measurement. Each chart contains hourly Tmrt data comparison for 3 days: clear sky, intermediate sky, and overcast sky, measured for the 3 different location zones (Figure 3 (a)). Measured Tmrt is calculated by the in-situ measurement of globe temperature $\left(\mathrm{T}_{\mathrm{g}}\right)$, air temperature $\left(\mathrm{T}_{\mathrm{a}}\right)$, wind velocity $\left(\mathrm{v}_{\mathrm{a}}\right)$ with our globe diameter dimension $(D: 0.15 \mathrm{~mm})$ and globe emissivity ( $\varepsilon: 0.95)$ in Equation (1) (Thorsson et al., 2007).

$$
\begin{gathered}
T_{m r t}=\left[\left(T_{g}+273,15\right)^{4}+1,1.10^{8} \cdot v_{a}^{0.6} \cdot\left(\varepsilon \cdot D^{0.4}\right)^{-1} \cdot\left(T_{g}-T_{a}\right)\right]^{0.25} \\
-273,15
\end{gathered}
$$

Chen et al. (2014) and Szucs et al. (2014) compared SOLWEIG, RayMan and ENVI-met based on Tmrt; however, these studies have evaluated the simulation values on the linear correlation with $\mathrm{R}^{2}$ values rather than absolute error measures such as RMSE and MBE.

The STEVE tool accuracy is based solely on the ambient air temperature comparison of the measured data with ENVI-met for Tmax, Tavg and Tmin values. The results demonstrate that ENVI-met has an acceptable range, as expected, the STEVE tool has a better agreement with RMSE are $0.34^{\circ} \mathrm{C}$ Tmin, $0.50^{\circ} \mathrm{C}$ Tavg, $0.88^{\circ} \mathrm{C}$ Tmax, and MBE -0.14 Tmin, $0.46 \mathrm{Tavg}, 0.77^{\circ} \mathrm{C}$ Tmax respectively (Figure 3 (b)). However, Figure 3 (b) illustrates that the STEVE tool overestimates Tmax and Tavg values and underestimates Tmin values. The reason could be the solar radiation impact on the STEVE regression model which is not included for Tmin while using daily maximum solar radiation value for Tmax. On the other hand, uncertainties in setting the specific humidity at 2,500 $\mathrm{m}$ value for ENVI-met might have a negative impact through to decrease the ambient air temperature.

The Tmrt results indicate that intermediate and overcast sky conditions have less predictive error than the clear sky condition. As Thorsson et al. (2007) tackled, RayMan mismatches results for early morning and evening time measurements due to low sun elevation as observed $\mathrm{C} 2$, C3, C4 location point in Figure 3(c). Although comparison is done with 24 hours/9 days data, the tools have a better agreement with the measurement results for daytime (7.00 am to $7.00 \mathrm{pm}$ ).

The PET comparison demonstrates that the prediction of thermal comfort by SOLWEIG is less accurate for the unshaded Greenery zone for intermediate and overcast sky regarding Tmrt accuracy as Szucs et al. (2014) mentioned in the studies. For instance, Figure 3(c) shows that SOLWEIG has a failure particularly on the G3 overcast sky plot with the most biased result RMSE 8.40 ${ }^{\circ} \mathrm{C}$ and MBE $-7.05{ }^{\circ} \mathrm{C}$. On the other hand, when SVF has a smaller value such as Passage zone the PET comparison illustrates better agreement for both SOLWEIG and RayMan.

As a consequence of the discussion section, assessed four tools have been developed aware of the need for environmental performance analysis to mitigate UHI and increase thermal comfort. This study interprets that a level of knowledge in the fields of urban planning (ENVI-met, SOLWEIG), human biometeorology or urban climatology (RayMan) is required to use the tools due to their complexity of geometric and physical input parameters. And also, even if these tools try to fill the gap between urban planning and meteorology experts and non-experts (STEVE tool), the scope of the tools might not be adequate for a comprehensive microclimate analysis. Linking between these research fields and microclimate studies, recent research has pointed out the need for mutual actions from architecture and urban planning remarkably helpful for 'urban design' (Lindberg et al., 2015). Considering that, the present study creates a framework for microclimate prediction tools by evaluating the usefulness of the tools from the point of a designer while handling the tools objectively to avoid presenting 'the best' tool. 

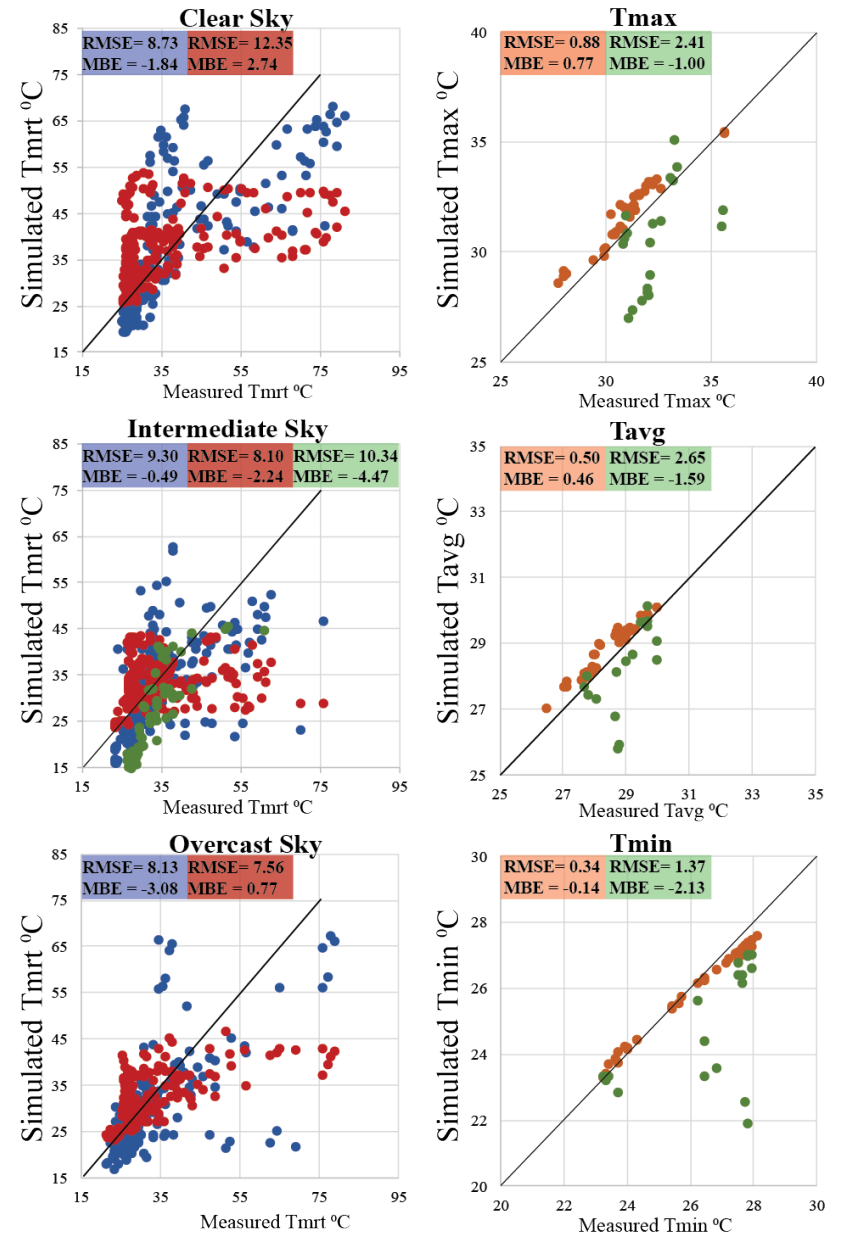

(a) Tmrt Comparison

(b) Tmax Tavg Tmin Comparison

SOLWEIG RayMan ENVI-met STEVE

\section{Conclusion}

This study comprehensively evaluates four urban microclimate tools-ENVI-met, RayMan, SOLWEIG, and STEVE-regarding their interfaces, advantages, disadvantages, limitations, capabilities, and accuracies on the prediction of air temperature, radiant temperature, and the thermal index PET. The comparisons are based on measurements and simulations during a 6 -week period in the tropical climate of Singapore.

The methodology employed provides a better understanding of each prediction tool. This study might be a document as a guide for the practical application of the four selected tools. According to the designer approach, scaling and detailing the model might influence the selection of microclimate prediction tool to obtain different results. The discussion section therefore indicates the capabilities and limitations of prediction tools abstracted by Table 2 .

The findings demonstrate the accuracy prediction results are in the acceptable range error on the results as seen Figure 3. The dominant difficulty of this study was the modelling of urban form in 4 different tools with 4 different methods. However, the evaluation of this study demonstrates the presence of a balance between advantages and disadvantages of the tools. While the

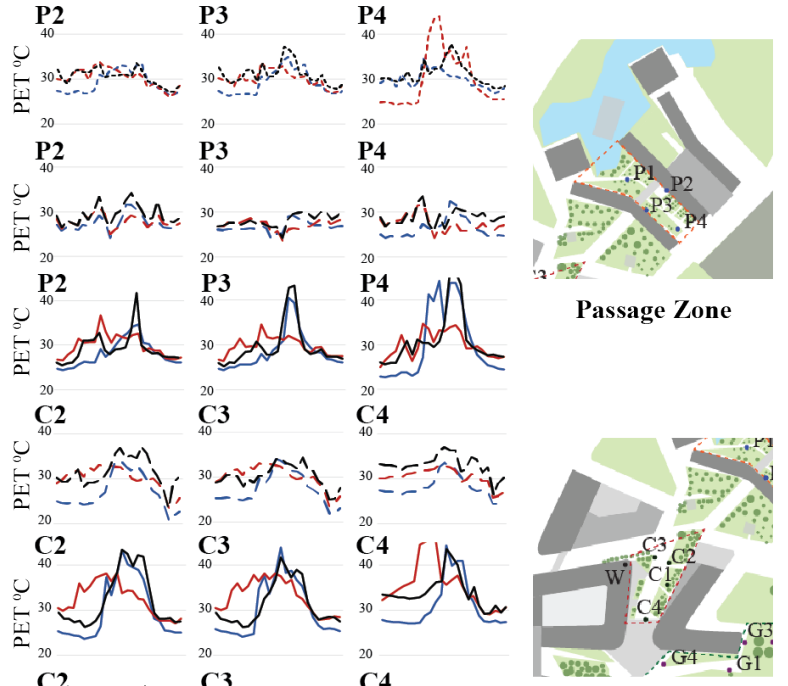

${ }_{0}^{C 2}{ }_{20}^{C 2}{ }_{40}^{C 3},{ }_{40}^{C 4}$

Courtyard Zone
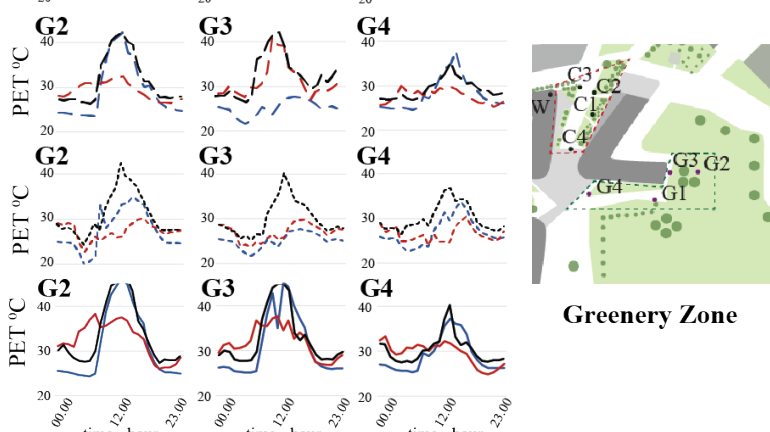

Greenery Zone

(c) PET Comparison

Clear Sky Intermediate Sky Overcast Sky SOLWEIG RayMan Measured

(b) is Tmax-Tavg-Tmin values; (c) is PET.

slowest tool is the most comprehensive one (ENVI-met) regarding the capabilities, the narrow scoped one (the STEVE tool) could provide the most accurate results. Nevertheless, from a view of an urban designer, a novel improvement is needed about the generating of an urban context model to evaluate environmental performance into tools.

For further studies, the scope would be extended by including CityComfort+ tool and by implementing the same method in the second university campus in Singapore to increase the sample points for accuracy.

The increasing concern of the microclimate predictions is a trending topic that needs the interdisciplinary approach by architects, designers, scientists and researchers, for the sake of mitigating UHI challenge and increasing thermal comfort. This comparison study, therefore, contributes an evaluation of the microclimate prediction tools to future users by an architect/researcher's point of view.

\section{Acknowledgement}

We would like to acknowledge the SUTD-MIT International Design Centre (IDC) for their support via the IDG21500108 project. This work supports the IDC's their grand challenge on Sustainable Build Environment. 


\section{References}

IPCC Report (2018). Summary for Policymakers of IPCC Special Report on Global Warming of $1.5^{\circ} \mathrm{C}$ approved by governments. Released Date: 8 October 2018: https://www.ipcc.ch/2018/10/08/summary-forpolicymakers-of-ipcc-special-report-on-globalwarming-of-1-5c-approved-by-governments/

Miguet, F., Groleau, D., \& Marenne, C. (1996). A combined sunlight and skylight tool for microclimatic analysis in urban architectures. In Solar energy in architecture and urban planning, Fourth European Conference. Berlin, March.

Bruse, M. and Fleer, H. (1998). Simulating surfaceplant-air interactions inside urban environments with a three dimensional numerical model. Environmental modelling \& software, 13(3-4), 373-384.

Matzarakis, A., Rutz, F., and Mayer, H. (2007). Modelling radiation fluxes in simple and complex environments - application of the RayMan model. International journal of biometeorology

Lindberg, F., Holmer, B., \& Thorsson, S. (2008). SOLWEIG 1.0-Modelling spatial variations of 3D radiant fluxes and mean radiant temperature in complex urban settings. International journal of biometeorology, 52(7), 697-713.

Jusuf, S. K., \& Wong, N. H. (2009). Development of empirical models for an estate level air temperature prediction in Singapore. In Proceedings of the Second International Conference on Countermeasures to Urban Heat Islands, Berkeley,(California) September.

Huang, J., Cede, J. G., and Spengler, J. D. (2014). CityComfort+: A simulation-based method for predicting mean radiant temperature in dense urban areas. Building and Environment, 80, 84-95.

Meteorological Service Singapore (2018). Climate of Singapore. http://www.weather.gov.sg/climateclimate-of-singapore/

Szucs, Á., Gál, T., \& Andrade, H. (2014). Comparison of measured and simulated mean radiant temperature. Case study in Lisbon (Portugal). Finisterra-Revista Portuguesa de Geografia, (98), 95-111.

Chen, Y. C., Lin, T. P., \& Matzarakis, A. (2014). Comparison of mean radiant temperature from field experiment and modelling: a case study in Freiburg, Germany. Theoretical and applied climatology

Ozkeresteci, I., Crewe, K., Brazel, A. J., \& Bruse, M. (2003). Use and evaluation of the ENVI-met model for environmental design and planning: an experiment on linear parks. In Proceedings of the 21st International Cartographic Conference (ICC), Durban, South Africa (pp. 10-16).

Wong, N.H., Ignatius, M., Eliza, A., Jusuf, S. K., and Samsudin, R. (2012). Comparison of STEVE and ENVI-met as temperature prediction models for Singapore context. International Journal of
Sustainable Building Technology and Urban Development, 3(3), 197-209.

Mayer, H., \& Höppe, P. (1987). Thermal comfort of man in different urban environments. Theoretical and applied climatology, 38(1), 43-49.

Lindberg, F., Grimmond, C., Onomura, S., Järvi, L., \& Ward, H. (2015). UMEP — an integrated tool for urban climatology and climate sensitive planning applications. In 9th Int. Conf. on Urban Climate.

EPW (2018), Energy Plus Weather Data by Location, Singapore. Access date: 20.05.2018. https://energyplus.net/weather-

location/southwest_pacific_wmo_region_5/SGP//SG P_Singapore.486980_IWEC

ASHRAE 55, (2004), Thermal Comfort chapter, Fundamentals volume of the ASHRAE Handbook, ASHRAE, Inc., Atlanta, GA.

NASA ACPC (2019) Atmospheric Correction Parameter Calculator. https://atmcorr.gsfc.nasa.gov/

Nastos, P. T., Vassilakis, E., Nastos, M. P. P., Charalampopoulos, I., \& Matzarakis, A. (2017). Assessment of continuous sky view factor based on ultra-high resolution natural colour images acquired by remotely piloted airborne systems for applications in an urban area of Athens. International journal of remote sensing, 38(20), 5814-5829.

Ignatius, M., Wong, N. H., \& Jusuf, S. K. (2015). Urban microclimate analysis with consideration of local ambient temperature, external heat gain, urban ventilation, and outdoor thermal comfort in the tropics. Sustainable Cities and Society, 19, 121-135.

Wessel, D. J. (2001). ASHRAE fundamentals handbook 2001 (SI Edition). American Society of Heating, Refrigerating, and Air-Conditioning Engineers, 31.

Höppe, P. (1999). The physiological equivalent temperature-a universal index for the biometeorological assessment of the thermal environment. International journal of Biometeorology, 43(2), 71-75.

Thorsson, S., Lindberg, F., Eliasson, I., \& Holmer, B. (2007). Different methods for estimating the mean radiant temperature in an outdoor urban setting. International journal of climatology, 27(14)

Salata, F., Golasi, I., de Lieto Vollaro, R., \& de Lieto Vollaro, A. (2016). Urban microclimate and outdoor thermal comfort. A proper procedure to fit ENVI-met simulation outputs to experimental data. Sustainable Cities and Society, 26, 318-343.

Blazejczyk, K., Epstein, Y., Jendritzky, G., Staiger, H., \& Tinz, B. (2012). Comparison of UTCI to selected thermal indices. International journal of biometeorology, 6(3), 515-53 